\title{
An Airfoil Shape Design using the Real-Coded Adaptive Range Multi-Objective Genetic Algorithm and PARSEC Method
}

\author{
SungKi Jung ${ }^{1}$ \\ Centro de Engenharia, Modelagem e Ciências Sociais, UFABC, Santo André, SP, \\ Luiz de Siqueira Martins Filho ${ }^{2}$ \\ Centro de Engenharia, Modelagem e Ciências Sociais, UFABC, Santo André, SP, \\ Fernando Madeira ${ }^{3}$ \\ Centro de Engenharia, Modelagem e Ciências Sociais, UFABC, Santo André, SP,
}

\begin{abstract}
Resumo. Trade-offs is one of important elements for engineering design problems characterized by multiple conflicting objectives that needs to be simultaneously improved. In this study, the real-coded Adaptive Range Multi-Objective Genetic Algorithm (ARMOGA) code, which represents the global multi-objective optimization algorithm, was developed for an airfoil shape design. In order to achieve the better aerodynamic characteristics than reference airfoil at landing and cruise conditions, maximum lift coefficient and lift-to-drag ratio were chosen as object functions. Futhermore, the PARSEC method reflecting geometrical properties of airfoil was adopted to generate airfoil shapes. Finally, two airfoils, which show better aerodynamic characteristics than a reference airfoil, were chosen. As a result, maximum lift coefficient and lift-to-drag ratio were increased of $4.89 \%$ and $5.38 \%$ for first candidate airfoil and $7.13 \%$ and $4.33 \%$ for second candidate airfoil.
\end{abstract}

Palavras-chave. Computational Fluid Dynamics (CFD), PARSEC, Adaptive Range MultiObject Genetic Algorithm (ARMOGA)

\section{Introduction}

The typical aerodynamic design process is an iterative process, requiring a number of design iterations to achieve balanced emphasis from the diverse inputs and

\footnotetext{
${ }^{1}$ sungki.jung@ufabc.edu.br,

2 luiz.martins@ufabc.edu.br

3 fernando.madeira@ufabc.edu.br
} 
outputs. To achieve an optimal design, researchers have developed optimization techniques, and research is actively being conducted on possible optimization method. In addition, industrial design problems in an aerospce engineering often have many design objectives with conflicting requirements. For example, high lift, low drag and weight are required to enhance the flight performance during the landing, take-off and cruise. These problems are typical Multi-Objective (MO) optimization problems. The aim of MO optimization is to determine a best design that satisfies requirements for each objective. A traditional way to meet the best design is based on a trade-off that is determined empirically or interactively by designers. It requires a time-consuming process in ordet to classify proper candidates in many solutions. Another attractive and alternative ways are to identify the proper candidates via trade-offs among multiple objectives and then select the best solution based on a suitable criterion. In the latter case, the trade-offs are represented as nondominated solutions, which are not dominated by any other solutions, called by the Pareto solutions. Ideally, Pareto solutions, which mean global non-dominated solutions that form global trade-offs, should be obtained to select the best solution from a set of non-dominated solutions. In addition, MO approach in stead of a single object optimization is not depended on the weight factors, which are usually applied in areas of single object optimization. According to the characteristics, the MO solutions are robust and engineers' intuitions on the weight factors can be sublated.

For many years, researchers have applied gradient-based optimization schemes to aerodynamic shape optimization [8]. Also, there has been growing interest in the use of global optimization methods in a wide range of design problems, as well as aerodynamic shape optimization. Hybrid optimization methods based on genetic and gradient search algorithms have been applied to wing planform[3]. Anderson et al. [1] applied Pareto genetic algorithms (GAs) to the multiobjective optimization of missile aerodynamic shape design. Tekinalp et al. [9] have developed a simulated annealing method for missile trajectory optimization.

In this study, the airfoil shape design to increase the lift and decrease the drag at specified angle of attack in which the maximum lift-to-drag ratio of a reference airfoil is shown has been conducted using Adaptive Range Multi-Object Genetic Algorithm (ARMOGA) proposed by Sasaki et al. [6] and PARSEC method, respectively. The ARMOGAs introduce the range adaptation, which changes the search region according to the statistics of better solutions. Here, a normal distribution is used to represent the design space efficiently, which was originally proposed by Arakawa et al. in binary-coded Adaptive Range Genetic Algorithms for single objective problem.[3] Oyama et al. extended the binary-coded to real-coded ARGAs[5] for design optimization. The ARMOGA is extended to MO optimization problems to treat multiple solutions and to maintain the diversity of solutions to collect multiple non-dominated solutions, unlike single objective problems. The PARSEC [7] airfoil generator based on the explicit mathematical functions are used for 2D curve definition for airfoil design which flowphenomena-oriented parameters control geometrical and aerodynamic properties. Airfoil shapes are blended with known analytical section formulae and generic variable camber wing sections are generated. Finally, the FLUENT, GAMBIT and TGRID are employed in order to design airfoil shapes using the Computational Fluid Dynamics (CFD) and aerodynamic grids. In addition, the ARMOGA and PARSEC codes are developed 
and an integrated system code which is consisted of the in-house and commericial codes is developed and shown in figure 1.

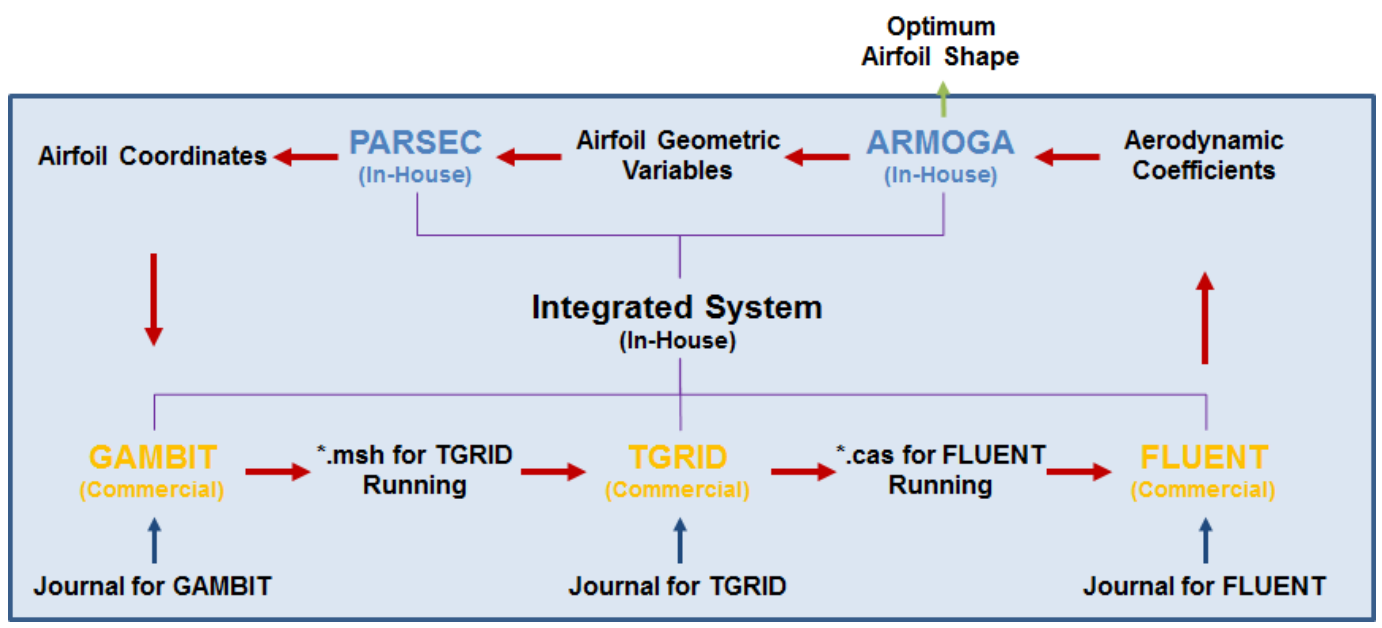

Figure 1: Integrated system and flow chart of optimization process using the CFD.

\section{Aerodynamics Airfoil Shape Design}

\subsection{Adaptive Range Multi-Object Genetic Algorithm}

The real-coded ARMOGA is based on the ARGA $[4,10]$ and most of process are exactly same with Muli-Objective Evolutionary Algorithm (MOEA). In general, evolutionary algorithms are based on the binary which is consisted of values of 0 and 1 in order to express the cromosome, ARMOGA however is consisted of the real number. In case of large value of design variables, the MOEA represented by the binary requires a mount of digits. As a consequense, the computer resources are heavily used. In adversely, the ARMOGA is more flexible for an allowance of computer resource, since all design variables are represented by real numbers. The main difference between the ARMOGA and the conventional MOEA is the introduction of the range adaptation. The essence of ARMOGAs is to adapt the population toward promising regions during the optimization process, which enables efficient and robust search in good precision while keeping the string length small. Moreover, ARMOGAs eliminate the need of prior definition of search boundaries since ARMOGAs distribute solution candidates according to the normal distributions of the design variables in the present population. The details of ARMOGA is described in reference [6].

\subsection{PARSEC Method}

PARSEC is very common and highly effective method of airfoil parameterization. It uses eleven basic parameters to completely define the aerofoil shape as shown in Figure 2. The various parameters are leading edge radius, upper crest location, lower crest location, upper and lower curvature, trailing edge coordinate and direction, trailing edge 
wedge angle and thickness. In this method, a linear combination of shape functions describes the aerofoil shape. With the help of defined geometric parameters, one can control the maximum curvature on upper and lower surfaces and their location, which greatly affect the occurrence of shock wave and its strength.

\subsection{CFD Solver}

In order to obtain the aerodynamic coefficients of candidated airfoils, the air flow should be computed using the CFD solver. The classical Reynolds-Averaged NavierStokes (RANS) equations were employed as the governing equations. The CFD solver is formulated for a finite control volume and solved using an explicit time marching procedure. For the spatial discretization Roe's approximate Riemann solver and Van Leer's monotone upstream-centred schemes (MUSCL) was employed. Van Albada's limiter was adopted in order to prevent the generation of oscillation and preserve the monotonicity. For the temporal discretization an implicit scheme was employed in order to accelerate the convergence. For the turbulence flow in flow fields, RANS was employed and the Spalart-Allmaras turbulent model was chosen to close the RANS. For the boundary conditions no-slip and Riemann invariant conditions were applied on the solid surface and the far-fields. An ideal gas equation was also employed to close the system of equations. A hibrid grid, which is consisted of the quadrature and triagonal grid for boundary layers and far field, respectively, are employed in order to capture the flow seperation near the solid surface and efficiently reduce the computation resource and calculation time.

\section{Application and Results}

In this study, the airfoil shape design to show the better aerodynamic performance than a reference airfoil is performed by the optimization process which includes the ARMOGA, PARSEC and CFD. Regarding the reference airfoil, one needs to know the aerodynamic characteristics of reference airfoil. Figure 2 shows the shape and aerodynamic characteristics of reference airfoil, respectively. The aerodynamic characteristics of reference airfoil is obtained by the CFD solver. In order to exactly capture viscous flow in boundary layer, $Y^{+} \sim 1.0$ and the height of first layer is 0.000183 percents of chord length and total number of layers is twenty-six. Flow conditions are Mach number 0.2 and 0.6, altitude, sea level and 25,000 ft and Reynolds numbers $1.1 \mathrm{e}+07$ and $1.8 \mathrm{e}+07$. Those conditions represent the landing and cruise conditions.

For the optimization of airfoil shape, the lift-to-drag ratio and maximum lift coefficient which represent the important aerodynamic factors for landing and cruise conditions are selected as object functions in equations (1). In addition, the object functions including max functions is proposed in order to utilize the previously obtained aerodynamics results of the reference airfoil. It can be considered as weight factors that efficiently search the dominant candidates among the whole airfoils. 

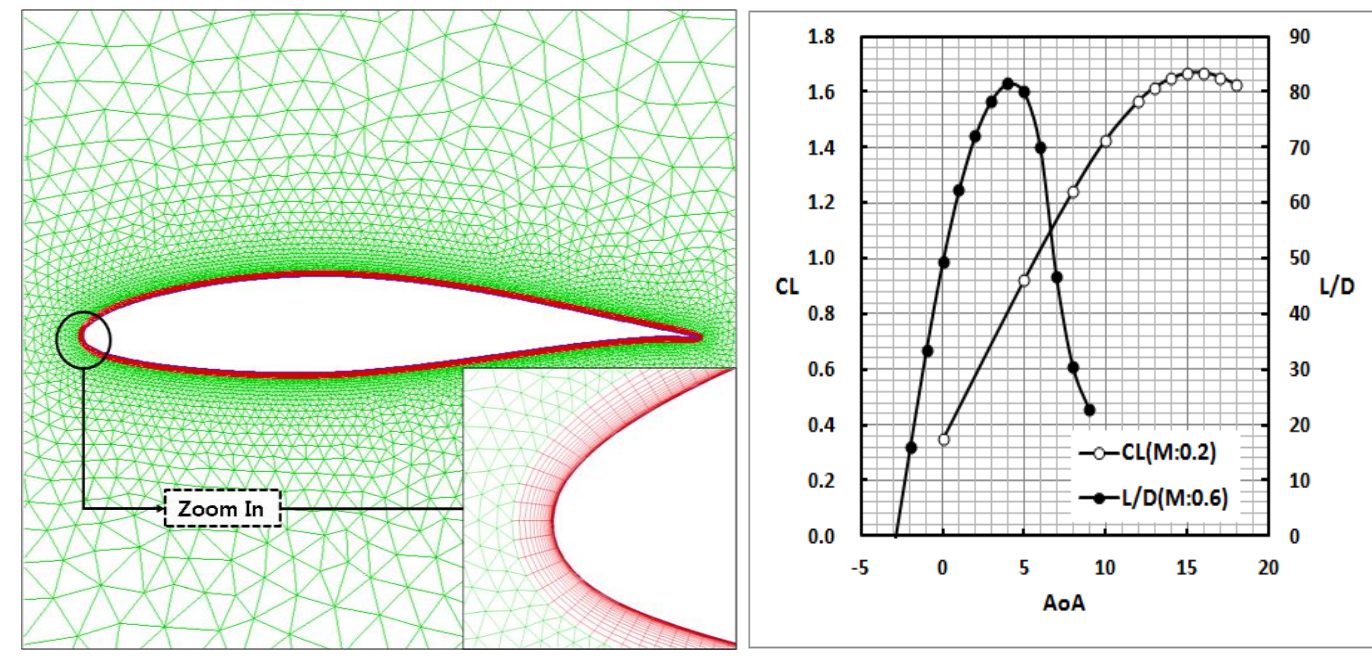

Figure 2: Reference airfoil (left) and its aerodynamic characteristics (right).

$$
\begin{aligned}
& \operatorname{Max} f_{1}(\mathbf{x})=\boldsymbol{L} / \boldsymbol{D}+\max \left\{\left[\boldsymbol{L} / \boldsymbol{D}-(\boldsymbol{L} / \boldsymbol{D})_{0}\right], 0\right\}, \\
& \operatorname{Max} \boldsymbol{f}_{2}(\mathbf{x})=\boldsymbol{C}_{\boldsymbol{L}_{\max }}+\max \left\{\left[\boldsymbol{C}_{\boldsymbol{L}_{\max }}-\boldsymbol{C}_{\left.\left.\boldsymbol{L}_{\max , 0}\right], 0\right\},}\right.\right.
\end{aligned}
$$

where, $(L / D)_{0}$ and $C L_{\max , 0}$ are maximum lift-to-drag ratio and lift coefficients of the reference airfoil at angles of attack, 4 and 16 degrees, respectively. Table 1 shows the optimization strategies based on the ARMOGA,

Tabela 1: Strageties of ARMOGA.

\begin{tabular}{|c|c|c|c|c|c|}
\hline Populations & Generations & Selection & Mutation & Crossover & Adaptive Range Generation \\
\hline 16 & 10 & Tournament & $4 \%$ & Uniform & 3rd \\
\hline
\end{tabular}

Figure 3 shows the optimization results. The $\mathrm{C} 1$ and $\mathrm{C} 2$ are final cadidated airfoils which show the better performances than the reference airfoil. At Mach 0.6, the weak shock wave around leading edge is observed, however the strength of shock waves of candidated airfoils are less than the reference airfoil. Figure 4 shows comparisons of shapes and aerodynamic characteristics among the candidated and reference airfoils. Even though the cambers of upper surfaces of candidated airfoils are slightly increased than the reference, the lift-to-drag ratios are higher than the reference airfoil. Even, it effects an increase of lift and drag, however the increase of lift-to-drag ratio is archieved by a reduction of the maximum thickness in order to decrease the drag. Drag divergence Mach numbers for each airfoil are almost the same and pitching moments show the negative. By the way, the reference airfoil shows more robust than candidated airfoils, however the difference among the airfoils are neglegible. As a result, maximum lift coefficient and lift-to-drag ratio were increased of $4.89 \%$ and $5.38 \%$ for first candidate airfoil and $7.13 \%$ and $4.33 \%$ for second candidate airfoil. 


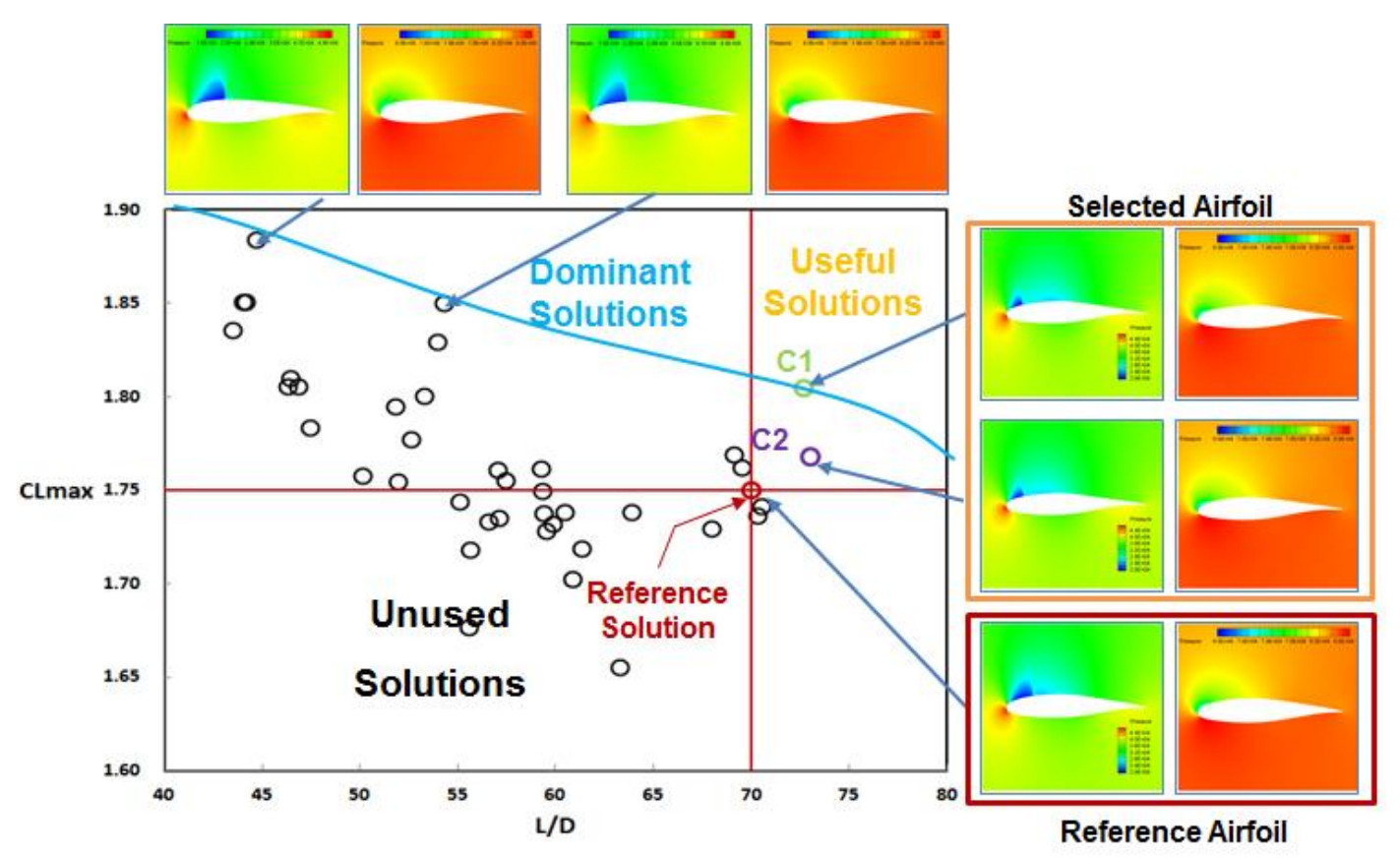

Figure 3: Dominant solutions and final candidated airfoils.
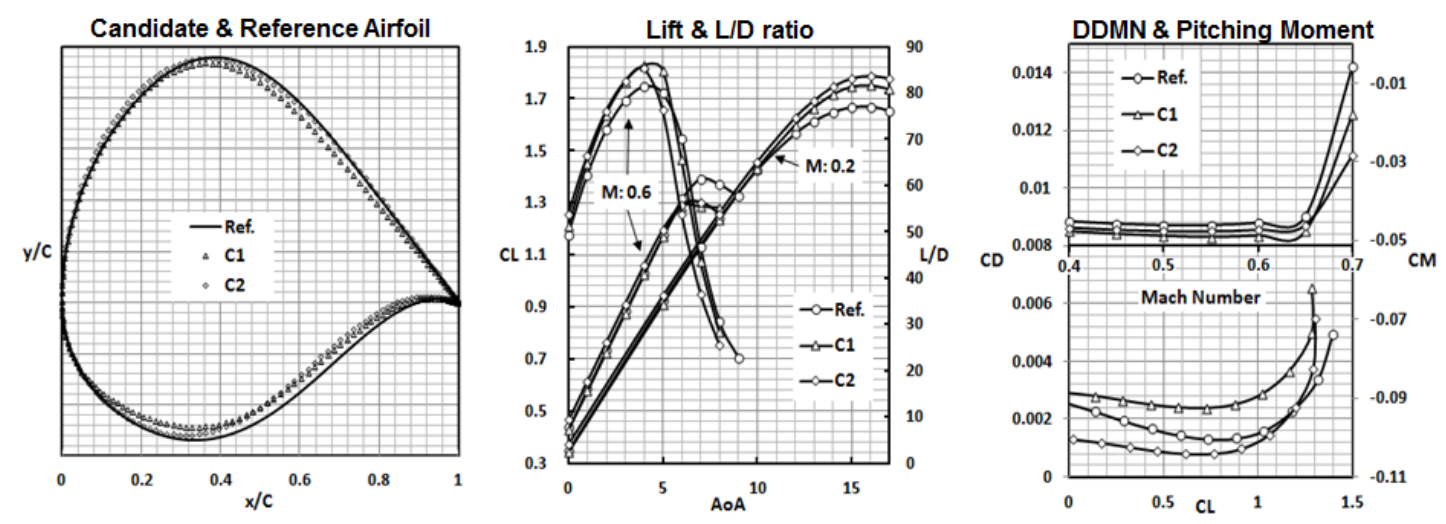

Figure 4: Candidated airfoil shapes and its aerodynamic characteristics.

\section{Conclusions}

In this study, aerodynamic shape design is conducted using in-house (ARMOGA and PARSEC) and commercial (FLUENT, GAMBIT and TGRID) codes. Candidates, $\mathrm{c} 1$ and c2, show better aerodynamic performance than reference airfoil. As a result, maximum lift coefficient and lift-to-drag ratio were increased of $4.89 \%$ and $5.38 \%$ for first candidate airfoil and $7.13 \%$ and $4.33 \%$ for second candidate airfoil. In the future, Self-Organizing Map will be applied in order to investigate a correlation between design variables and object functions. 


\section{References}

[1] M. B. Anderson, J. E. Burkhalter and R. M. Jenkins, Missile Aerodynamic Shape Optimization Using Genetic Algorithms, Journal of Spacecraft and Rockets, vol. 37, 663-669, (2000).

[2] M. Arakawa and I. Hagiwara, Nonlinear Integer, Discrete and Continuous Optimization Using Adaptive Range Genetic Algorithms, Proceedings of 1997 ASME Design Engineering Technical Conferences, (1997).

[3] N. F. Foster and G. S. Dulikravich, Three-Dimensional Aerodynamics Shape Optimization Using Genetic and Gradient Search Algorithms, Journal of Spacecraft and Rockets, vol. 34, 36-42, (1997).

[4] S. K. Jung, R. S. Myong and T. H. Cho, An Efficient Global Optimization Method for Reducing the Wave Drag in Transonic Regime, Journal of the Korean Society for Aeronautical and Space Sciences, vol. 37, 248-254, (2009).

[5] A. Oyama, S. Obayashi and T. Nakamura, Real-Coded Adaptive Range Genetic Algorithm Applied to Transonic Wing Optimization, Applied Soft Computing, vol. 1, 179-187, (2001).

[6] D. Sasaki and S. Obayashi, Efficient Search for Trade-Offs by Adaptive Range Multi-Objective Genetic Algorithms, Journal of Aerospace Computing, Information, and Communication, vol. 2, 45-64, (2005).

[7] H. Sovieczky, Parametric airfoils and Wings, Notes on Numerical Fluid Mechanics, Vieweg, 71-88, (1998).

[8] O. Tanrilulu and V. Ercan, Optimal External Configuration Design of Unguided Missiles, Journal of Spacecraft and Rockets, vol. 35, 312-316, (1998).

[9] O. Tekinalp and M. Bingol, Simulated Annealing for Missile Optimization: Developing Method and Formulation Techniques, Journal of Guidance, Control, and Dynamics, vol. 27, 616-626, (2004).

[10] Y. R. Yang, S. K. Jung, T. H. Cho and R. S. Myong, Aerodynamic Shape Optimization System of a Canard-Controlled Missile Using Trajectory-Dependent Aerodynamic Coefficients, Journal of Spacecraft and Rockets, vol. 49, 243-249, (2012). 